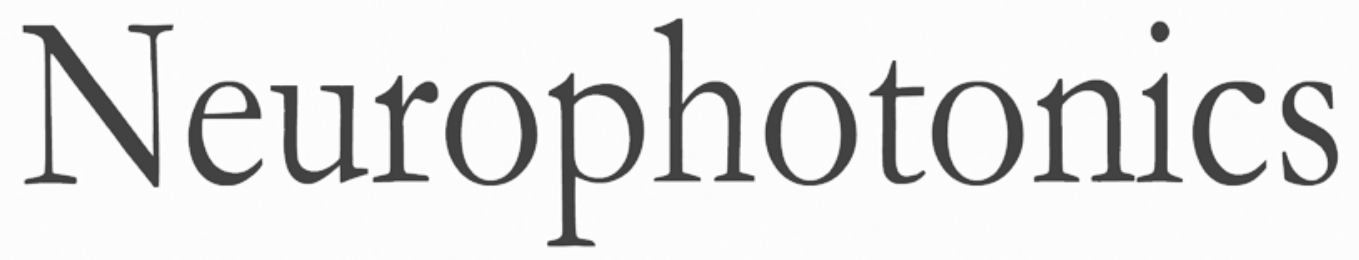

\title{
Optimized multimodal functional magnetic resonance imaging/near- infrared spectroscopy probe for ultrahigh-resolution mapping
}

Lia Maria Hocke Kenroy Cayetano

Yunjie Tong

Blaise Frederick 


\title{
Optimized multimodal functional magnetic resonance imaging/near-infrared spectroscopy probe for ultrahigh-resolution mapping
}

\author{
Lia Maria Hocke, ${ }^{\mathrm{a}, \mathrm{b}, \mathrm{k}}$ Kenroy Cayetano, ${ }^{\mathrm{a}, \mathrm{c}}$ Yunjie Tong, ${ }^{\mathrm{a}, \mathrm{c}}$ and Blaise Frederick ${ }^{\mathrm{a}, \mathrm{c}}$ \\ ${ }^{a}$ McLean Hospital, McLean Imaging Center, 115 Mill Street, Belmont, Massachusetts 02478, United States \\ ${ }^{\mathrm{b} T}$ Tufts University, Department of Biomedical Engineering, 4 Colby Street, Medford, Massachusetts 02155, United States \\ 'Harvard Medical School, Department of Psychiatry, 25 Shattuck Street, Boston, Massachusetts 02115, United States
}

\begin{abstract}
Functional near-infrared spectroscopy (fNIRS) is an increasingly important noninvasive method in neuroscience due to its high temporal resolution and ability to independently measure oxy- and deoxy-hemoglobin. However, the relatively low spatial resolution of fNIRS makes it difficult to relate this signal to underlying anatomy. Simultaneous functional magnetic resonance imaging (fMRI) can complement fNIRS with superior spatial resolution and the ability to image the entire brain, providing additional information to improve fNIRS localization. However, current simultaneous fMRI/fNIRS acquisition methods are not optimal, due to the poor physical compatibility of existing MR coils and fNIRS optodes. Here, we present a technique to manufacture a true multimodal fMRI/fNIRS probe in which both modalities can be used with maximal sensitivity. To achieve this, we designed custom MR coils with integral fNIRS optodes using three-dimensional printing. This multimodal probe can be used to optimize spatial $(1.2 \times 1.2 \times 1.8 \mathrm{~mm})$ and temporal resolution $(2.5 \mathrm{~Hz})$ of fMRI, and it provides maximal MRI sensitivity, while allowing for high flexibility in the location and density of fNIRS optodes within the area of interest. Phantom and human data are shown to confirm the improvement in sensitivity in both modalities. This probe shows promise for addressing fundamental questions of the relation of fNIRS to physiology. $\odot$ 2015 Society of Photo-Optical Instrumentation Engineers (SPIE) [DOI: 10.1117/1.NPh.2.4.045004]
\end{abstract}

Keywords: optics; functional near-infrared spectroscopy; functional magnetic resonance imaging; ultra-high spatial resolution; ultrahigh temporal resolution; simultaneous measurements.

Paper 15030RR received Jun. 3, 2015; accepted for publication Oct. 29, 2015; published online Dec. 10, 2015.

\section{Introduction}

Functional near-infrared spectroscopy (fNIRS) ${ }^{1}$ has become a widely used noninvasive measurement technique to investigate brain functions, along with techniques such as electroencephalography and functional magnetic resonance imaging (fMRI). It detects changes in light absorption and scattering in tissue caused by changes in concentration of oxy- $(\Delta[\mathrm{HbO}])$ and deoxy-hemoglobin $(\Delta[\mathrm{Hb}])$, thought to indirectly reflect neural activity through neurovascular coupling. The most commonly used form of fNIRS is continuous wave (CW) fNIRS. ${ }^{2}$ Major technical advantages of CW fNIRS include high temporal resolution $(\sim 25$ to $100 \mathrm{~Hz}$ ) of hemoglobin concentration measurements and the ability to distinguish between oxy- and deoxy-hemoglobin. Major challenges include (1) relatively poor spatial resolution (1 to $3 \mathrm{~cm}^{3}$ ) caused by scattering of light in tissue and the geometry of the light sources and detectors on the surface of the head, (2) the limited penetration depth $\left(1 \mathrm{~cm}\right.$ of cerebral cortex $\left.{ }^{3}\right)$, and (3) the fact that data measured are a mixture of signals coming from various layers of the head, including skin, scalp, superficial layers, and cortex. ${ }^{4-7}$ Various studies have tried to address the sensitivity of fNIRS to these superficial layers. ${ }^{8-13}$ Techniques such as those we developed here, which directly map the neuronal and physiological sources observed by fNIRS, can be highly advantageous for the field of fNIRS.

*Address all correspondence to: Lia M. Hocke, E-mail: lia.hocke@ucalgary.ca
One technique used to improve fNIRS localization is to employ simulations to derive a more accurate sensitivity distribution by using a physically realistic photon propagation model. Simulations are based on the finite element method $^{14,15}$ or Monte Carlo method. ${ }^{16}$ This can include using realistic head templates in the Monte Carlo simulation. The results support general assumptions about fNIRS sensitivity, such as that larger source-detector separation leads to increased sensitivity toward brain activation farther from the scalp. ${ }^{17-20}$ In addition, they show the strong contribution of the scalp to fNIRS signals. ${ }^{21}$ However, while this does refine localization, these simulations are still static representations of the brain and do not account for physiological contributions. Recently, it has been shown that the fNIRS signal is influenced even more by non-neuronal physiological interferences than previously thought. ${ }^{13}$

In addition to simulation, concurrent fMRI/fNIRS studies are widely used to assess sensitivity of the fNIRS signal, because fMRI has high spatial resolution ( 2 to $3 \mathrm{~mm}$ ) with wholebrain coverage (for a review, see Ref. 22). These studies include sensitivity adjustments to the fNIRS profiles ${ }^{23}$ as well as measurements of specific influences to fNIRS, including the influences from extra-cerebral layers, ${ }^{24}$ cerebral blood flow, ${ }^{25}$ extra-cranial impact thought to come from skin veins, ${ }^{12,13}$ and pial veins. ${ }^{11}$ However, all described concurrent studies face physical challenges to combine fMRI and NIRS (Fig. 1 shows that with current dense phased array fMRI coils, little 


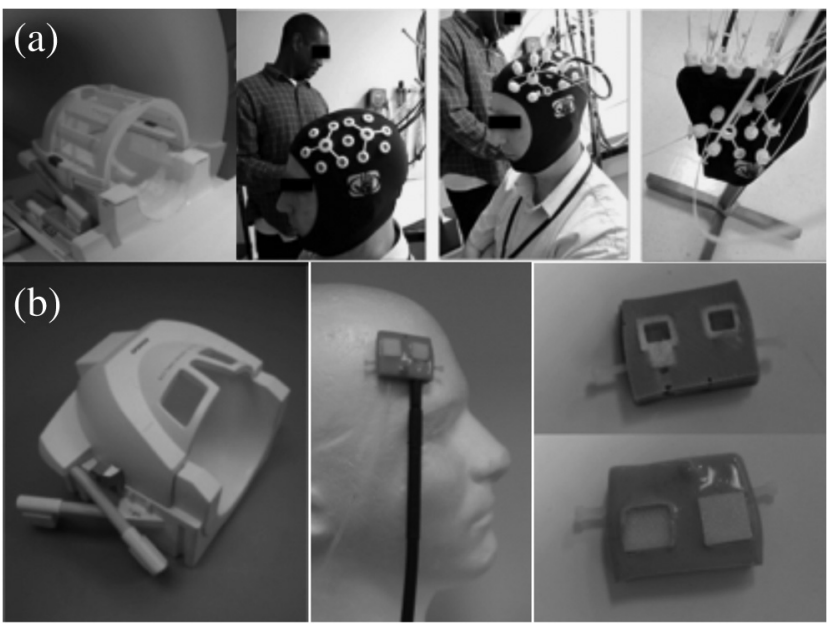

Fig. 1 Higher sensitivity of the MR coils leads to MR coil designs which leave nearly no space for functional near-infrared spectroscopy (fNIRS) optodes. (a) The Siemens 12-channel MR coil is depicted [left panel in (a)]. A functional magnetic resonance imaging (fMRI)-compatible probe designed to fit into the coil is shown in the right three pictures. (b) However, to take advantage of the current standard for $\mathrm{fMRI}$ acquisition, especially its high spatial resolution, coils as in the Siemens 32-channel phased array coil [left panel in (b)] are manufactured. These are closer to the head, leaving little to no space for fNIRS optodes to be placed. The coil leaves $\sim 5 \mathrm{~mm}$ space between the head and coil in the front. This leaves only the possibility to compromise highly on the location and density of fNIRS optodes as seen in our previous design, in which we used right-angle prisms machined out of plexiglas, which could be squeezed below a small part of the frontal lobe.

space is available for NIRS optodes), which leads to compromises in one or both modalities, such as (1) low fMRI spatial resolution $\left(\sim 3 \times 3 \times 3 \mathrm{~mm}^{11-13,23-25}\right)$, which prevents the clear separation of extra-cerebral layers due to insufficiently small voxel size to separate signals in these narrow areas; additionally, the low spatial resolution and sensitivity can lead to a failure to detect small activation patterns, such as when using older, less powerful coil arrays (e.g., the study by Kirilina et al. ${ }^{12}$ is the only one mentioned above that used the newest 32-channel coil instead of less powerful coils; however, as a result, she did not actually conduct simultaneous measurements but separated NIRS and fMRI measurements); (2) low fMRI temporal resolution, which results in aliasing of higher-frequency signals in fMRI, such as cardiac and respiratory signals, confounding activation patterns [e.g., all mentioned studies acquired data at $\sim 2 \mathrm{~s}$ repetition time (TR $)^{11-13,23-25}$; ; (3) the lack of flexibility in the positioning of the fNIRS probe to any desired location; (4) limitations on the density of fNIRS source-detector pairs, which is critical for depth sensitivity; (5) poor coupling of the fNIRS optodes to the scalp in the fNIRS probe, which leads to reduced sensitivity and more motion artifacts; and (6) limited accuracy in matching the fNIRS optode locations to fMRI [e.g., Gagnon et al. ${ }^{11}$ used vitamin E fiducial markers, which can result in location inaccuracies of as much as 2 to $3 \mathrm{~mm}$ (Ref. 26)]. Resolving these problems will permit experiments that can not only deepen our understanding of the sources of fNIRS signals and lead to better interpretation of the fNIRS signal, but also help us to validate and develop methods, such as the use of different source-detector distances, ${ }^{27}$ aiming to remove extra-cerebral noise.

In this study, we address the above-mentioned problems by developing a true multimodal probe (Fig. 2). With the design of this probe, we introduce three concepts: (1) we manufacture our own MR coil to fit fNIRS; (2) we introduce three-dimensional (3-D) printing technology to print the fMRI/fNIRS probe with unprecedented flexibility for the density and location of fNIRS optodes in the critical area and the volume covered with fMRI; (3) we introduce area-specific probe making, which can ultimately be extended to subject-specific probe designs, for maximum sensitivity and data quality.

\section{Methods}

\subsection{Probe Design}

Unlike previous fMRI/fNIRS studies where fNIRS optodes are made to fit the MR coil, in this probe, the MR coil is designed to

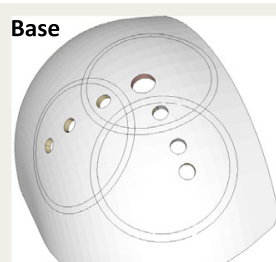

(a)

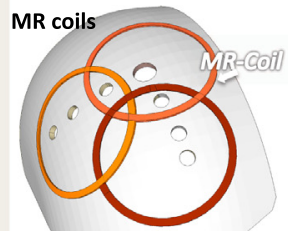

(b)

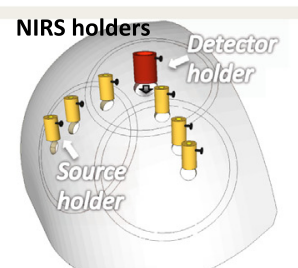

(c)

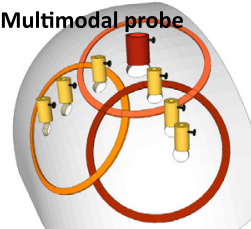

(d)

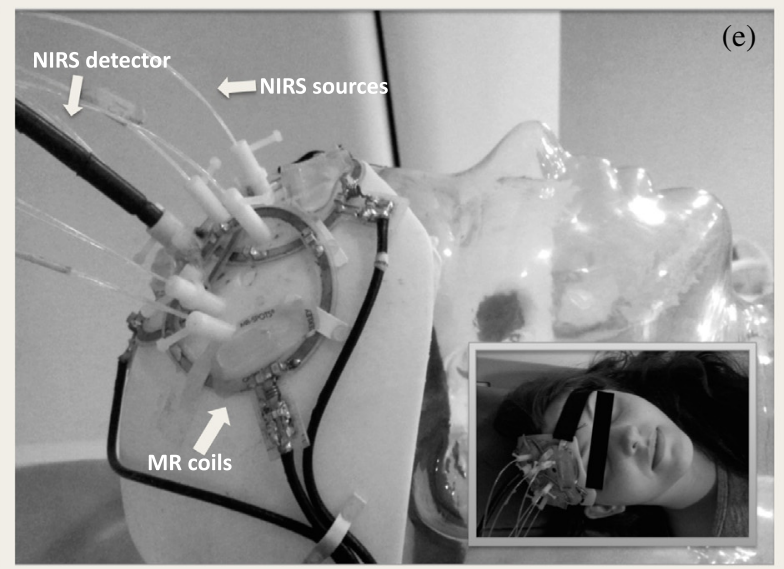

Fig. 2 Multimodal probe design. The various parts of the multimodal probe are shown. (a) First the base can be printed with the 3-D printer. (b) Next, MR coils were manufactured and fixed on the base (MR coils). (c) As a last step, matching fNIRS fiber holders were printed, which fit snugly into the holes in the base (NIRS holders). (d) The combination of all parts can be seen as in the Google Sketchup diagram, which was used to generate the final printed objects (multimodal probe). (e) Its realistic view on a phantom and on a participant lying in the scanner (indented picture on the right). 
fit the fNIRS optodes. Since we are only interested in the voxels in the vicinity of the fNIRS probe, we abandoned whole-brain coverage in exchange for high spatial and temporal resolution in a restricted region, here the prefrontal cortex. This region was selected for two reasons. (1) The prefrontal cortex is a region frequently imaged in clinical studies of mental disorders, so we chose a region for which subsequent results would have high implications in the field. (2) Activation in the prefrontal cortex is more localized than that of motor activation and therefore a good candidate to test the spatial specificity of the probe.

\subsubsection{MR coil design}

As stated above, our goal was to design a probe in which the MR coil is designed to fit the fNIRS optodes. A number of criteria had to be taken into account in order to achieve high spatial and temporal resolution. Spatial resolution in fMRI is a function of many parameters, including field of view (FOV), number of voxels across the FOV, sampling bandwidth, number of phase encode lines, and the total amount of time allotted for recording a slice, all of which interact. Moreover, the slice acquisition time determines the number of slices which can be recorded within a given repetition time (thus setting the ultimate temporal resolution of the fMRI acquisition). Therefore, the smaller the number of slices acquired per repetition, the higher the possible temporal resolution of fMRI.

In practice, we can stack multiple acquisition blocks and reduce the FOV in the phase encode direction to decrease the slice acquisition time and, hence, increase the temporal resolution but also preserve spatial coverage. ${ }^{28}$ As an alternative (but only when available in the facility), we can use the multiband sequence acquisition. ${ }^{29,30}$ We limited the imaging volume to a $10 \times 10 \mathrm{~cm}^{2}$ area with a phased array of three RF coils in order to enhance the signal-to-noise ratio (SNR) at high spatial resolution and capture only a focal area (here the prefrontal cortex). ${ }^{31,32}$ The RF coils were fixed to a base [Fig. 2(b)] (printed on a 3-D printer with acrylonitrile butadiene styrene (ABS) material, uPrint SE 3D, Stratasys, Eden Prairie, Minnesota), which was shaped to the prefrontal area of the head. Because 3-D printing allows printing of arbitrary shapes, shaping the base of the probe to a high-resolution anatomical image of the specific participant's head is straightforward; however, for the coil (first generation) used in this study, we physically measured the curvature of the subjects' forehead and fitted an approximate geometrical form to it in Google SketchUp ${ }^{\mathrm{TM}}$. This was done to prove the concept and simplify the procedure. In the future, we will use a standard brain template [i.e., MNI152 (Montreal Neurological Institute)] and the subject's anatomical scan to maximize the applicability of the probe. The 3-D printed base [Fig. 2(a)] fits to the shape of the scalp over the prefrontal cortex, which results in close and stable contact of the coil and the head. This is important in order to decrease motion artifacts and maximize received signal strength at the cortical surface.

The circular coils had a diameter of $\sim 5 \mathrm{~cm}$ resulting in a penetration depth of $\sim 2.5 \mathrm{~cm}$. Because fNIRS is unable to detect signal deeper than $\sim 2 \mathrm{~cm}$ beneath the skull, this radius was chosen to give the smallest coil diameter which would image the sensitive region of fNIRS at high-fMRI SNR. The receive-only MR coils were tuned to $123.25 \mathrm{MHz}$ to match the center frequency of the Siemens 3T. We used geometrically orthogonal coils in the almost Olympic configuration ${ }^{31-33}$ in order to minimize mutual inductance [Fig. 2(b)]. Additional decoupling was accomplished using preamp decoupling (a low-input impedance preamplifier is transformed to a high impedance at the coil terminals with a quarter wave cable to isolate the various elements of an array). The coil was interfaced to the scanner through a four-channel preamplifier interface (Stark Contrast, Erlangen, Germany).

In summary, we developed a phased array with three overlapping RF coils in an equilateral triangle shape, which were mounted on a base shaped to the desired area of the head, here the prefrontal cortex.

\subsubsection{Integration of functional near-infrared spectroscopy probes}

The design of the fNIRS probe is of critical importance in the study in order to ensure good contact with the skin (high SNR), avoid motion artifacts, ensure subject comfort, and allow for the desired spatial coverage and depth sensitivity. To achieve these, different criteria (other than those for the MR coil) have been considered. We decided to incorporate at least six source-detector pairs (SD1 to SD6) with different source-detector separations (two detectors with three sources each at 2-, 3-, and 4$\mathrm{cm}$ separation), to assess the depth sensitivity of fNIRS and cover most of the area of interest. In the example study described below, we used exactly six sources and one detector, resulting in six NIRS channels (each source-detector pair is defined as an NIRS channel). The probe is capable of accommodating more channels. The holders for the fNIRS detector and source fibers [Fig. 2(c)] are made with the 3-D printer. The printing material is ABSplus plastic, which is nonferromagnetic and does not cause susceptibility artifacts in the fMRI data. The material is also lightweight, which ensures the subject's comfort. In addition, we can control the thickness and color of the material used in the probe, which can block or absorb the external light. In this study, we used white ABSplus plastic, and given the size and position of the region of interest (ROI), a quadrant of an oblate spheroid was chosen, with a thickness of $2.4 \mathrm{~mm}$ and the following internal dimensions: an equatorial radius of $10.2 \mathrm{~cm}$ and a semiminor radius of $7.62 \mathrm{~cm}$.

The probes were directly integrated into the base onto which the coils were fixed to ensure stability and avoid motion artifacts [Fig. 2(c)]. This integration was achieved by drilling (or printing) holes into the base between the coils. These cylindrical hollow tubes (probe holders) allow optical fibers to be freely adjusted to ensure good contact with the skin. There are two reasons for making the fNIRS holders separate from the base. The first is to make adjustment of the hair below the sources and detector fibers easy. These holes were snug enough that we could place the cylindrical probe holders in them without additional fastening mechanism. Through the holes, hair could be shifted out of the probe area beforehand. The second reason was to improve the quality of the probe. 3-D printed materials have different strengths along different axes. By printing the base and the holders separately, we were able to maximize the strength of each component.

An additional problem in simultaneous fMRI and fNIRS studies is the precise registration of fNIRS optode locations with the MR data to accurately determine the sensitive region of the fNIRS measurements. Normally, localization measurements such as the 10-20 system or vitamin E MR markers are used, with clear limitations. The former is known to be a rough estimate, and the latter lacks accuracy due to the vitamin capsule size $(\sim 10 \times 5 \times 5 \mathrm{~mm})$ and the chemical shift image displacement of the lipid contents of the capsule. ${ }^{26} \mathrm{We}$ 
incorporated MR visible markers into the probe by filling tygon tubing with dilute solution of a gadolinium MR contrast agent (Prohance) in water. The tube's diameter was $\sim 3 \mathrm{~mm}$, and it was wrapped around all NIRS optodes to make them visible in both the structural and functional MR images (not shown). We used the marker's location on the higher structural scans to locate the optodes and registered them (using FSL toolbox img2imgcoord) to the functional scans.

\subsection{Data Acquisition}

To rigorously test the performance of the fMRI component of the multimodal probe, we tested its performance against the Siemens 32-channel phased array coil. Both phantom as well as human data were taken for complete comparison. To show an example of the potential of the combination of fNIRS and fMRI with high spatial and temporal resolution, we also conducted a task-evoked paradigm study in a single subject. The McLean Hospital institutional review board approved all human experimental acquisitions, and participants were compensated for their participation.

\subsubsection{Magnetic resonance imaging signal-to-noise ratio}

For the comparison between the SNR of the 32-channel coil and the multimodal probe, data were acquired from a biomedical informatics research network phantom ${ }^{34}$ and from a participant lying quietly in the scanner. All MR data were acquired on a Siemens TIM Trio 3 Tesla whole-body MR scanner. Multiband echo planar imaging (EPI) data were obtained (University of Minnesota sequence cmrr_mbep2d_bold R011 ${ }^{29,30}$ ) with the following parameters: $\mathrm{TR} / \mathrm{TE}=525 / 30 \mathrm{~ms}$, flip angle $66 \mathrm{deg}$, matrix $96 \times 96$ on a $172 \times 172 \mathrm{~mm}$ FOV, multiband factor $=$ $3,24 \times 1.8 \mathrm{~mm}$ slices with no gap. Slices were prescribed tangent to the surface of the head at the location of the receiving fibers. We acquired 720 time points (378 s).

\subsubsection{Validation experiment}

For the task-evoked paradigm experiment, functional data from the multimodal probe were acquired for three tasks: (1) a rapid semantic task, (2) a null or control task for the rapid semantic task, and (3) a subtraction task, which was repeated twice. The tasks were partly replicated from earlier studies. ${ }^{12}$ The duration of the first, rapid semantic task block was $15 \mathrm{~s}$, during which abstract or concrete words were presented for $1 \mathrm{~s}$ with $1 \mathrm{~s}$ interstimulus interval, resulting in eight stimuli in one block. The participant had to press the right button when an abstract word preceded a concrete word (e.g., for "Thought"+"Cup" or "Liberty"+"Pen"). Otherwise, the participant had to press the left button. The control task was identical in all regards, except that the right button was pressed only if a specifically determined word combination occurred (e.g., every time "Cup" preceded "Thought"). The semantic and control tasks were separated by a $30 \mathrm{~s}$ rest period. The subtraction task consisted of two blocks of $15 \mathrm{~s}$ separated by a $20 \mathrm{~s}$ rest period, where a pair of numbers was shown on the monitor-a large number followed by a smaller one, which had to be sequentially subtracted from the first number (e.g., 365-13). The participant was reminded each time to breath normally. The entire run of all tasks ( $30 \mathrm{~s}$ rest, $15 \mathrm{~s}$ semantic task, $30 \mathrm{~s}$ rest, $15 \mathrm{~s}$ null semantic task, $30 \mathrm{~s}$ rest, two blocks of $15 \mathrm{~s}$ subtraction separated by $20 \mathrm{~s}$ ) lasted $192 \mathrm{~s}$ and was repeated five times.
Magnetic resonance imaging data acquisition. A highresolution anatomical image was obtained using the body coil in order to give uniform intensity images for registration [MPRAGE, repetition time/inversion time/echo time $(\mathrm{TR} / \mathrm{TI} / \mathrm{TE})=2530 / 1100 /[3.31,6.99,8.85,10.71], 256 \times 256 \times$ 128 voxels over a $256 \times 256 \times 170 \mathrm{~mm}$ sagittal slab, generalized autocalibrating partially parallel acquisition factor of $2,1 \times$ $1 \mathrm{~mm}$ in plane isotropic with slice thickness of $1.33 \mathrm{~mm}]$. For the functional acquisition, we deviated from the above-mentioned protocol to show that even without advanced acquisition sequences as the multiband sequence, data quality can be as high or higher in spatial and temporal resolution and provides meaningful data, which can be replicated in any facility. Afterward, we serially imaged multiple adjacent subvolumes (segments) of the brain. ${ }^{28}$ Each segment consisted of a stack of five $1.8 \mathrm{~mm}$ consecutive slices with no gap. For each acquisition, segments were acquired parallel to the surface of the coil (from only the scalp layer toward further inside the brain), and each acquisition was a stimulus-evoked scan lasting $192 \mathrm{~s}$ with the following parameters. EPI data were obtained with the following parameters: $\mathrm{TR} / \mathrm{TE}=400 / 30 \mathrm{~ms}$, flip angle $42 \mathrm{deg}$, matrix $128 \times 64$ on a $150 \times 75 \mathrm{~mm}$ FOV. Slices were prescribed perpendicular to the surface of the head in an oblique plane between the transverse and coronal planes at the location of the receiving fibers. We acquired 480 time points per stack with a brief pause $(\sim 10 \mathrm{~s})$ between each consecutive stack scan. All acquisitions were performed twice, once with the multimodal coil, and once with the Siemens 32-channel phased array.

fNIRS data acquisition. fNIRS data were recorded continuously during, and for several minutes before and after, the fMRI acquisition. The optical device used in this experiment was a near-infrared tissue imager (Imagent, ISS, Champaign, Illinois) comprising 16 laser sources at 690 and $830 \mathrm{~nm}$ (average power of $\sim 1 \mathrm{~mW}$ ) and four optical detectors (photomultiplier tubes, Hamamatsu Photonics R928). The imager was placed in the control room, and 10-m-long optical fibers (illumination fibers with $400 \mu \mathrm{m}$ core diameter) and fiber bundles (a bundle of $50 \mu \mathrm{m}$ fibers $3 \mathrm{~mm}$ in diameter) were used to connect the laser diodes and detectors with optical probes in the $3 \mathrm{~T}$ scanner. The fibers were made of glass with plastic at the end of the detector fiber (high susceptibility artifacts were not found). Measurements were acquired from six source-detector pairs. A detector was one collection fiber, and a source was a pair of illumination fibers ( 690 and $830 \mathrm{~nm}$ ). The separation between the collection and illumination fibers was 2,3 , and $4 \mathrm{~cm}$ along two lines at $90 \mathrm{deg}$ to each other. The sampling rate of the fNIRS data acquisition was $6.25 \mathrm{~Hz}$, as predetermined by the ISS instrument for the source-detector configuration. The probe geometry is shown in Fig. 2(c).

\subsection{Analysis}

\subsubsection{Magnetic resonance imaging signal-to-noise ratio}

MRI data were processed in FSL FEAT [FMRIB Expert Analysis Tool, v5.0.7 (Ref. 35), Oxford University, United Kingdom). ${ }^{36}$ For the comparison of the SNR between the coils, standard preprocessing steps were applied to the data: temporal high-pass filter (cut-off frequency $=0.01 \mathrm{~Hz}$ to remove very slow instrumental drifts) and motion correction (FSL FLIRT ${ }^{37,38}$ ). In the beginning of the temporal time course, 20 volumes were discarded to allow for T1 relaxation. In order 
to calculate the SNR, an ROI was chosen, and the mean intensity of the signals divided by the mean standard deviation of the signals at each time point was calculated over a sphere of $7 \mathrm{~mm}$ ( $\sim 59$ voxels). In addition, the mean intensity of the signals divided by the mean standard deviation over the entire time course over the same region was calculated to estimate temporal SNR. SNR and temporal SNR are separate measures of the coils' spatial and temporal performance, which we can compare between coils. An additional preprocessing step for the temporal standard deviation was to smooth voxels with a $1.8 \mathrm{~mm}$ kernel. Because of the geometry of the multimodal probe, the SNR advantage relative to the 32-channel coil will be greatest at the scalp. For the human participant, the anatomical voxel used was visually selected in the center of the most sensitive area of the multimodal probe (centered $\sim 2 \mathrm{~cm}$ from the surface of the head), to make the best estimate of the effective SNR. This is close to the maximum depth of penetration for fNIRS, and as the B1 sensitivity of the multimodal coil is much less uniform than that of the 32-channel coil in the ROI used, this comparison will tend to favor the 32-channel coil. We were unable to make comparisons with only a subset of the 32-channel coils due to the algorithmically selected coils (including remote ones) with multiband.

\subsubsection{Validation experiment}

For the fMRI data of the task-evoked study, each of the five fMRI slice stacks was preprocessed as described above. In addition, data were prewhitened and the five preprocessed fMRI slice stacks were concatenated in the slice direction (fslmerge) prior to a general linear model analysis. The stimulation protocol was convolved with the hemodynamic response function. The significance threshold at the voxel level was set to $z=2.3$ with a cluster threshold of $p=0.05$ (applied to the clusters remaining after the local voxel threshold). The fMRI data were also coregistered to the paricipant's structural scan, where fNIRS markers are visible. Moreover, we calculated fNIRS sensitivity profiles below these source-detector pairs in order to analyze the spatial overlap between fMRI and fNIRS data. The photon probability densities were calculated using an updated version of a locally written program to calculate the Banana path using the models described in Ref. 39. The original version of this software was used in the analyses in Ref. 23. Briefly, the user specifies the coordinates on the neuroimaging informatics technology initiative anatomic image where the source and detector fibers are placed. The software finds the tangent plane to the head at each fiber location and constructs a plane which intersects the line between the two points and is perpendicular to each of the tangent planes. The line between the source and detector is the $x$ axis, and the line perpendicular to the $x$ axis lying in the constructed plane is the $z$ axis. The probability density is then calculated using Eq. (13) from Ref. 39.

Each pair of raw fNIRS time courses (690- and 830-nm data) was converted into two time courses representing oxy$(\Delta[\mathrm{HbO}])$ and deoxy-concentration change $(\Delta[\mathrm{Hb}])$ using the modified Beer-Lambert law ${ }^{40,41}$ in MATLAB ${ }^{\circledR}$ (The Mathworks, Natick, Massachusetts). For the differential path length factors, we used published values of 6.51 at $690 \mathrm{~nm}$ and 5.86 at $830 \mathrm{~nm}^{42}$ The low-frequency component $(0.01$ to $0.2 \mathrm{~Hz})$ of $\Delta[\mathrm{HbO}]$ and $\Delta[\mathrm{Hb}]$ was obtained by using a zero-delay Fourier-domain band-pass filter in MATLAB ${ }^{\circledR}$. The low cutoff was chosen to adapt the frequency region in which the activation was expected. The fNIRS data corresponding to the five fMRI scans were extracted into separate time courses. The individual fNIRS time courses were then detrended, despiked, motion corrected, ${ }^{43}$ and normalized (divided by their standard deviation). Folding averages (an average over the identical periods of a task paradigm) for all task periods were calculated by averaging over all five repetitions of the task. We compared the average time course to the task paradigm by convolving the task paradigm with the hemodynamic response function.

\section{Results}

\subsection{Magnetic Resonance Imaging Signal-to-Noise Ratio}

The SNR of the signals acquired by the multimodal probe and the 32-channel coil in both phantom and human data are shown in Fig. 3. In both cases, the SNR of the multimodal probe exceeded that of the 32-channel coil in the ROI (Fig. 3).

\subsubsection{Probe functional magnetic resonance imaging signal-to-noise ratio test-phantom}

For the phantom data (Fig. 3, left panels), we found an SNR of $366.33 \pm 2.73$ for the multimodal probe compared to an SNR of $259.43 \pm 4.64$ for the 32-channel coil, an improvement of $41 \%$. The temporal SNR was 248.75 for the multimodal probe and 116.68 for the 32 -channel coil, a $113 \%$ improvement.

\subsubsection{Probe functional magnetic resonance imaging signal-to-noise ratio test-human data}

When the probe was tested on a human subject (Fig. 3, right panel), we found an SNR of $61.74 \pm 3.73$ for the multimodal probe and an SNR of $29.70 \pm 1.87$ for the 32-channel coil in the same cortical region, a $108 \%$ improvement. The temporal SNR was 57.85 for the multimodal probe and 28.55 for the 32-channel coil, a 103\% improvement.

\subsection{Validation Experiment}

The results of the fMRI data in the task-evoked experiment show the effect of the high data quality for the multimodal probe fMRI data (Fig. 4), both in the spatial $(1.2 \times 1.2 \times 1.8 \mathrm{~mm})$ as well as the temporal domain $(2.5 \mathrm{~Hz})$. Tasks are shown in green (rapid semantic task), yellow (control task for rapid semantic task), and blue (subtraction task). Brain activations of all tasks were detected with statistical significance (for the rapid semantic task, the control task, and the subtraction task, $z=9.94$,

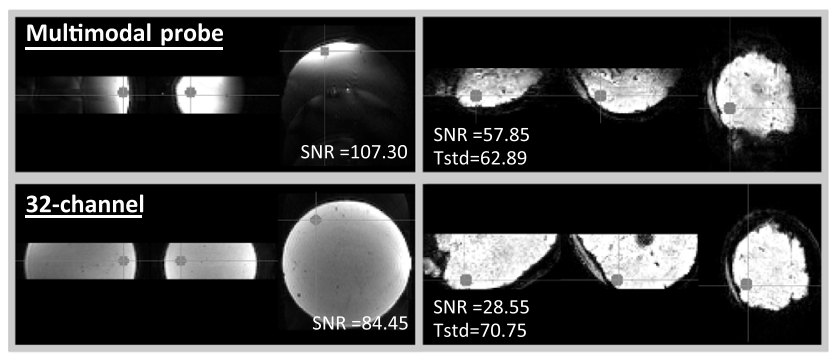

Fig. 3 Multimodal probe acquires data with superior signal-to-noise ratio (SNR). To compare quality performance of the MRI component of the multimodal probe, the SNR and the temporal SNR were calculated for the latest Siemens coils, the 32-channel MR coil, on a phantom (left panels) as well as a human participant (right panels). 


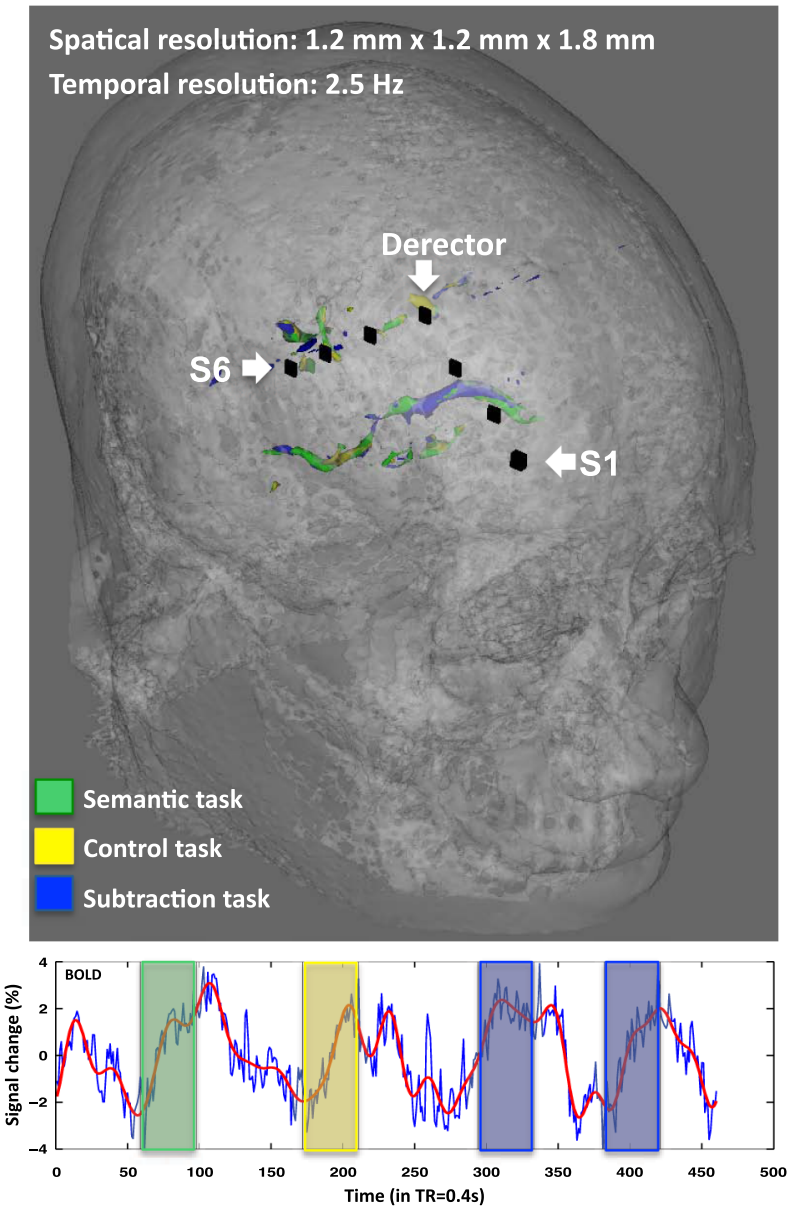

Fig. 4 Maximal sensitivity of fMRI. Activation patterns of the different tasks are shown in green (rapid semantic task), yellow (control task), and blue (subtraction task). fNIRS optode locations are shown as black diamonds (sources 1 and 6 are marked with $\mathrm{S} 1$ and S6). In addition, an example BOLD time course is depicted (lower panel) in which all activations are visible. Original data were plotted in blue, overlaid by a low-pass filtered version in red to show the response to the tasks. Task blocks were overlaid with their corresponding color.

8.64 , and 11.55 , respectively). These localized small activation patterns below the fNIRS probes (Fig. 4, black diamonds mark position of fNIRS optodes) are visible for all tasks. An example BOLD time course of one voxel is shown (Fig. 4, lower panel), in which all three tasks are visible. Original data were plotted in blue, showing the cardiac signal, overlaid by a low-pass filtered version in red to show the brain response to the tasks. Task blocks were overlaid with their corresponding color.

fNIRS data were high in quality with clearly visible cardiac pulsations in each channel. The results for the fNIRS data in the task-evoked paradigm experiment in Fig. 5 show the advantage of the high flexibility and density of fNIRS probes. Three source-detector pairs (SD1 to SD3) are depicted. The density and accurate location of fNIRS source-detector pairs allowed us to both capture the brain activation and separate the signal contributions from different depths [Fig. 5(b), yellow-red]. The fMRI activation patterns for the three different tasks were spatially merged into one activation pattern for all tasks [Fig. 5(a), green]. This was only done for visualization purposes as they overlapped below the fNIRS probes SD1 to SD3 (also see Fig. 4). Folding averages of the fNIRS $\Delta[\mathrm{Hb}]$ data over five trials are depicted in Fig. 5(b). Task activation patterns are clearly visible in the long-distance measurements SD3 $(3 \mathrm{~cm})$. The correlation between the NIRS time courses (from SD3 and SD1) and the hemodynamic convolved stimulation paradigm was $r=0.29$ and $r=0.77$, respectively. The greater the spatial overlap between the location of the MRI activation pattern and the sensitivity profile of the fNIRS data, the more pronounced the task-activation pattern in the fNIRS data itself.

The powerful combination of optimized sensitivity and simultaneous recording of fMRI and fNIRS is shown in Fig. 6 . Time courses of the fNIRS $\Delta[\mathrm{Hb}]$ and fNIRS $\Delta[\mathrm{HbO}]$ folding averages over five trials for SD1 [Fig. 6(a)] and SD6 [Fig. 6(b)] (both $4 \mathrm{~cm}$ source-detector pair separation) are depicted. In addition, enlarged fMRI activation patterns below the source-detector pairs from Fig. 4 are shown. The MR activation patterns differed greatly from each other, especially in their magnitude. The activation patterns below SD6 were so small that standard fMRI acquisition might not even have detected them at all, especially when smoothing clustered voxels. In fNIRS data, although task activation was visible in $\Delta[\mathrm{Hb}]$ in both source-detector pairs, there was a notable difference in the fNIRS $\Delta[\mathrm{HbO}]$ task response. SD1 showed an increase in fNIRS $\Delta[\mathrm{HbO}]$ for each task block, while SD6 did not. This distinction would not be possible with fMRI alone as it cannot independently measure oxy- and deoxy-hemoglobin, demonstrating that combining these modalities has the potential to enhance our understanding of brain activation.

\section{Discussion}

In this study, we present an integrated multimodal probe, constructed with purpose-built MR coils and a 3-D printed frame to simultaneously measure fNIRS and fMRI signals. We developed an MRI coil array, which we optimally tuned with high spatial resolution $(1.2 \times 1.2 \times 1.8 \mathrm{~mm})$ and temporal resolution $(2.5 \mathrm{~Hz})$ on a restricted FOV. We fixed these coils on a 3-D printed base, shaped to the head, and directly integrated fNIRS optodes into the design, allowing for stable contact of the fNIRS probes to the head. The 3-D printed design allowed for flexibility in the location and density of the fNIRS optodes, even in the center of the MRI coils, maximizing the spatial resolution of fMRI signals directly at the fNIRS optode locations. Moreover, we used gadolinium-doped markers to accurately register fNIRS optode locations. This allowed for a truly optimized multimodal measurement, which is a powerful tool for neuroimaging.

\subsection{Simple and Cheap Fabrication}

The design we show is easy to fabricate and relatively inexpensive. Fabrication of the base through 3-D printing allows for a flexible design, which can be easily replicated. The 3-D printed base is a simple shape, which can be rendered to any desired form. This can optimize the probe to different locations on the head and can even customize it to a participant's head for longitudinal studies. The reconfiguration of the probe only requires printing a new base. The entire probe base can be printed in under $5 \mathrm{~h}$, and the materials cost is under $\$ 10$. Most of the radiofrequency parts are reusable between probes (they are affixed to the base with screws), so reconfiguring the probe uses under $\$ 10$ in materials. In total, the entire probe costs around $\$ 1000$ when made from scratch without the preamplifier, which can be purchased separately and can be reused for additional probes. 

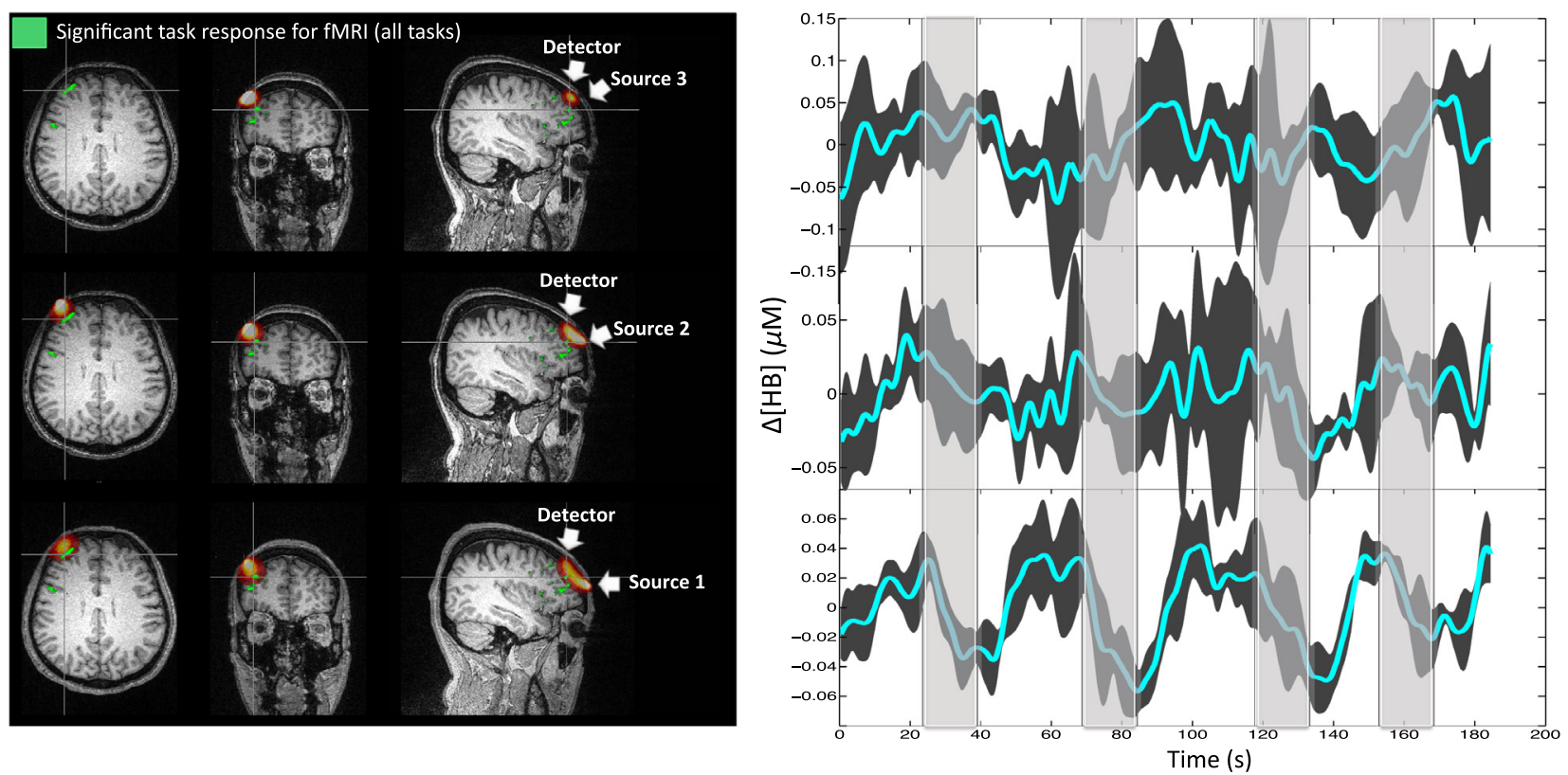

Fig. 5 Maximization of flexibility in fNIRS location and density. (a) fMRI task activation patterns of all tasks combined (green) and sensitivity profiles of three source-detector pairs (SD1 to SD3) with 4-, 3-, and 2-cm source-detector separation (red-yellow), respectively. (b) Corresponding folding average of the fNIRS $\Delta[\mathrm{Hb}]$ data over five trials. Gray overlaid bars indicate the activation blocks.
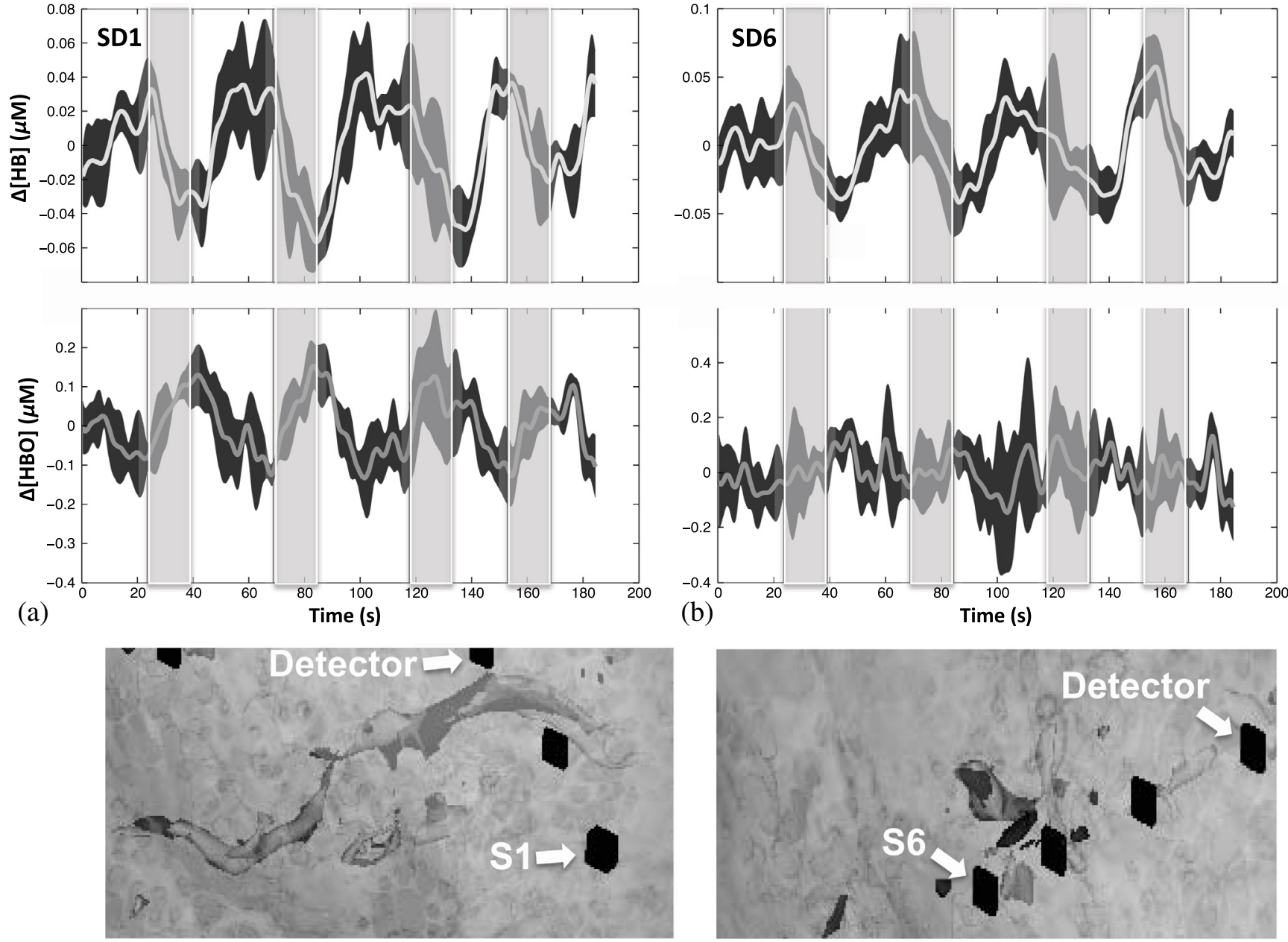

Fig. 6 Powerful fNIRS-fMRI combination to distinguish activation patterns. fNIRS $\Delta[\mathrm{Hb}]$ and $\Delta[\mathrm{HbO}]$ folding averages over five trials for (a) source-detector pair 1 (SD1) and (b) source-detector pair 6 (SD6) are depicted. Gray overlaid bars indicate the activation blocks. In addition, the corresponding enlarged fMRI activation patterns below the source-detector pairs from Fig. 4 are depicted. 


\subsection{High-Quality Data for Both Functional Near-Infrared Spectroscopy and Functional Magnetic Resonance Imaging}

Both fMRI and fNIRS data were of high quality. In our area of interest, the SNR of the multimodal probe exceeded that of a Siemens 32-channel phased array coil, which is the current standard for fMRI acquisition, both in phantom as well as human brain data (Fig. 3). Small activation patterns $(\sim 3 \mathrm{~mm})$ are localized, and moreover, the overlapping patterns could be resolved (Fig. 4). These extremely small differences in activation patterns would have been lost with the bigger voxel size required in most concurrent fMRI studies. In addition, even one of the fastest physiological signals, the cardiac signal $(\sim 1 \mathrm{~Hz})$, could be measured directly in fMRI, allowing the separation of the relevant task-related signal from confounding signals through simple spectral filtering, without aliasing of these signals as is usually seen in fMRI studies. Equally important, the multimodal probe allowed for high-quality fNIRS data in all the channels. This is achieved by the matching contours between the multimodal probe shape and the head, which gave the best optical coupling between the head and the fNIRS source and detectors.

\subsection{Sensitivity of Functional Magnetic Resonance Imaging}

This optimized multimodal measurement allows us to address questions regarding the spatial sensitivity of fNIRS signals with much higher accuracy. This is a difficult problem as the fNIRS signal comes from different layers within the head, and separating these layers is not easy with typical fMRI acquisition. Various methods have been employed to address this question as discussed in Sec. 1. Moreover, empirical approaches such as multidistance measurements ${ }^{44,45}$ are being applied on the basis of these sensitivity profiles, ${ }^{27,46}$ in particular to capture and remove the fNIRS signal contributions from the superficial layer. These methods also need to be validated.

With concurrently acquired fMRI data with superior spatial resolution, the multimodal probe allows us to address the question of NIRS sensitivity without relying on probabilitybased models regarding the path of the photon propagation. We know exactly where the fNIRS optodes are in relation to the changes in each layer below the optodes with $1.2 \times 1.2 \times$ $1.8 \mathrm{~mm}^{3}$ spatial resolution. In Fig. 5, we demonstrate that the short distance measurement, although spatially positioned above the activation, cannot detect the activation due to its depth in accordance with the sensitivity profile of the fNIRS. We also show that this changes when longer measurements are taken and the depth of the fNIRS sensitivity profile increases. In this longer source-detector separation measurement, the activation pattern is detected. There is even a visible difference between the fNIRS signal of $3 \mathrm{~cm}(r=0.29$ with task paradigm $)$ and $4 \mathrm{~cm}$ ( $r=0.77$ with task paradigm), showing that the more the activation location is within the sensitivity profile of the fNIRS, the better the activation is detected. These results are in accordance with the photon-propagation models described before and previous findings showing high spatial overlap between the fMRI and NIRS activation patterns, ${ }^{23,24,47}$ supporting these results. However, these studies also showed small variations for different tasks at different locations between fMRI and fNIRS activation patterns, which could not be explained. ${ }^{24}$ With the multimodal probe, we might be able to reevaluate these differences and determine the precise overlap between the modalities. The observations, although only from one participant, demonstrate the potential of this unique probe to address one of the major questions in the fNIRS field; this is the subject of a study currently underway in our laboratory.

\subsection{Oxy- and Deoxy-Hemoglobin Measurement}

Another fundamental question in the fNIRS field is the spatial sensitivity to oxy- and deoxy-hemoglobin. Task-specific fNIRS changes were often detected by both oxy- and deoxy-hemoglobin, but sometimes only by one of them. ${ }^{24}$ Moreover, spatial discongruencies between these species were found. ${ }^{24,47}$ It is hypothesized that differences between the hemoglobin measurements stem from differences in susceptibility to local versus global changes. fNIRS $\Delta[\mathrm{HbO}]$ is thought to be more closely related to cerebral blood volume and global effects, ${ }^{48}$ whereas fNIRS $\Delta[\mathrm{Hb}]$ is more closely linked to regional activity. ${ }^{49}$

We showed in Fig. 6 that the small and spatially scattered brain activations (reflected in the fMRI map) can only be detected by the fNIRS $\Delta[\mathrm{Hb}]$ signal, while relatively large and clustered brain activations can be detected by both fNIRS $\Delta[\mathrm{HbO}]$ and $\Delta[\mathrm{Hb}]$ signals. This observation is consistent with previous findings. Moreover, due to the high spatial resolution of our data and accurate localization of the fNIRS probe, we can specify and quantify the conditions (e.g., size of the activation pattern) in which this observation is true and try to understand the physiology underlying it.

In summary, we have manufactured and validated a new true multimodal probe and have demonstrated the potential of this unique probe to address fundamental questions in the fNIRS field. As stated in Sec. 2, we abandoned whole-brain coverage in order to maximize sensitivity and spatial and temporal resolution in a restricted region. This limits the applicability of this probe for resting state studies. This work is primarily for the comparison between fNIRS and fMRI and the investigation of the sensitivity of fNIRS, for which a restricted region is sufficient. As more data are collected, we hope to have a more thorough understanding of the sensitivity and specificity of the fNIRS signal.

\section{Acknowledgments}

We thank Dr. Scott Lukas, Dr. Sinem Erdogan, and Dr. Kimberly Lindsey for their help. This work was supported by the National Institutes of Health R21 DA027877 and R21 DA032746. We have no conflicts to declare.

\section{References}

1. F. F. Jobsis, "Noninvasive, infrared monitoring of cerebral and myocardial oxygen sufficiency and circulatory parameters," Science 198(4323), 1264 (1977).

2. F. Scholkmann et al., "A review on continuous wave functional nearinfrared spectroscopy and imaging instrumentation and methodology," Neuroimage 85(Pt 1), 6-27 (2014).

3. D. A. Boas et al., "Improving the diffuse optical imaging spatial resolution of the cerebral hemodynamic response to brain activation in humans," Opt. Lett. 29(13), 1506-1508 (2004).

4. A. Young et al., "Behaviour of near-infrared light in the adult human head: implications for clinical near-infrared spectroscopy," $\mathrm{Br} . \mathrm{J}$. Anaesth. 84(1), 38 (2000).

5. S. L. Davis et al., "Skin blood flow influences near-infrared spectroscopy-derived measurements of tissue oxygenation during heat stress," $J$. Appl. Phys. 100(1), 221 (2006).

6. T. J. Germon et al., "Extracerebral absorption of near infrared light influences the detection of increased cerebral oxygenation monitored 
by near infrared spectroscopy," J. Neurol. Neurosurg. Psychiatry 58(4), 477-479 (1995)

7. P. W. McCormick et al., "Intracerebral penetration of infrared light," J. Neurosurg. 76(2), 315-318 (1992).

8. N. M. Gregg et al., "Brain specificity of diffuse optical imaging: improvements from superficial signal regression and tomography," Front. Neuroenerget. 2(14), 1-8 (2010).

9. I. Tachtsidis et al., "False positives in functional near-infrared topography," Adv. Exp. Med. Biol. 645, 307-314 (2009).

10. F. Tian et al., "Enhanced functional brain imaging by using adaptive filtering and a depth compensation algorithm in diffuse optical tomography," IEEE Trans. Med. Imaging 30(6), 1239-1251 (2011).

11. L. Gagnon et al., "Quantification of the cortical contribution to the NIRS signal over the motor cortex using concurrent NIRS-fMRI measurements," Neuroimage 59(4), 3933-3940 (2012).

12. E. Kirilina et al., "The physiological origin of task-evoked artifacts in functional near infrared spectroscopy," in Proc. of the 17th Annual Meeting of the Organization for Human Brain Mapping, Quebec City (2011).

13. E. Kirilina et al., "Identifying and quantifying main components of physiological noise in functional near infrared spectroscopy on the prefrontal cortex," Front. Hum. Neurosci. 7, 864 (2013).

14. S. R. Arridge et al., "A finite element approach for modeling photon transport in tissue," Med. Phys. 20(2 Pt 1), 299-309 (1993).

15. A. H. Hielscher, R. E. Alcouffe, and R. L. Barbour, "Comparison of finite-difference transport and diffusion calculations for photon migration in homogeneous and heterogeneous tissues," Phys. Med. Biol. 43(5), 1285-1302 (1998).

16. L. Wang, S. L. Jacques, and L. Zheng, "MCML-Monte Carlo modeling of light transport in multi-layered tissues," Comput. Methods Programs Biomed. 47(2), 131-146 (1995).

17. G. E. Strangman, Q. Zhang, and Z. Li, "Scalp and skull influence on near infrared photon propagation in the Colin27 brain template," Neuroimage 85(Pt 1), 136-149 (2014).

18. Y. Hoshi et al., "Reevaluation of near-infrared light propagation in the adult human head: implications for functional near-infrared spectroscopy," J. Biomed. Opt. 10(6), 064032 (2005).

19. C. Mansouri et al., "Depth sensitivity analysis of functional near-infrared spectroscopy measurement using three-dimensional Monte Carlo modelling-based magnetic resonance imaging," Lasers Med. Sci. 25(3), 431-438 (2010).

20. T. Li, H. Gong, and Q. Luo, "Visualization of light propagation in visible Chinese human head for functional near-infrared spectroscopy," J. Biomed. Opt. 16(4), 045001 (2011).

21. J. Selb et al., "Sensitivity of near-infrared spectroscopy and diffuse correlation spectroscopy to brain hemodynamics: simulations and experimental findings during hypercapnia," Neurophotonics 1(1), 015005 (2014).

22. J. Steinbrink et al., "Illuminating the BOLD signal: combined fMRIfNIRS studies," Magn. Reson. Imaging 24(4), 495-505 (2006).

23. A. Sassaroli et al., "Spatially weighted BOLD signal for comparison of functional magnetic resonance imaging and near-infrared imaging of the brain," Neuroimage 33(2), 505-514 (2006).

24. X. Cui et al., "A quantitative comparison of NIRS and fMRI across multiple cognitive tasks," Neuroimage 54(4), 2808-2821 (2011).

25. T. J. Huppert et al., "A temporal comparison of BOLD, ASL, and NIRS hemodynamic responses to motor stimuli in adult humans," Neuroimage 29(2), 368-382 (2006).

26. D. Agrawal and P. Steinbok, "Fiducials: Achilles' heel of image-guided neurosurgery: an attempt at indigenization and improvement," Clin. Neurosurg. 56, 80-83 (2009).

27. L. Gagnon et al., "Short separation channel location impacts the performance of short channel regression in NIRS," Neuroimage 59(3), 25182528 (2012).

28. Y. Tong and B. Frederick, "Concurrent fNIRS and fMRI processing allows independent visualization of the propagation of pressure waves and bulk blood flow in the cerebral vasculature," Neuroimage 61(4), 1419-1427 (2012).

29. D. A. Feinberg et al., "Multiplexed echo planar imaging for sub-second whole brain FMRI and fast diffusion imaging," PLoS One 5(12), e15710 (2010).
30. S. Moeller et al., "Multiband multislice GE-EPI at 7 tesla, with 16 -fold acceleration using partial parallel imaging with application to high spatial and temporal whole-brain fMRI," Magn. Reson. Med. 63(5), 11441153 (2010).

31. B. Keil and L. L. Wald, "Massively parallel MRI detector arrays," J. Magn. Reson. 229, 75-89 (2013).

32. L. L. Wald et al., "Phased array detectors and an automated intensitycorrection algorithm for high-resolution MR imaging of the human brain," Magn. Reson. Med. 34(3), 433-439 (1995).

33. L. L. Wald et al., "Proton spectroscopic imaging of the human brain using phased array detectors," Magn. Reson. Med. 34(3), 440-445 (1995).

34. L. Friedman and G. H. Glover, "Report on a multicenter fMRI quality assurance protocol," J. Magn. Reson. Imaging 23(6), 827-839 (2006).

35. http://www.fmrib.ox.ac.uk/fsl

36. S. M. Smith et al., "Advances in functional and structural MR image analysis and implementation as FSL," Neuroimage 23(Suppl 1), S208-219 (2004).

37. M. Jenkinson et al., "Improved optimization for the robust and accurate linear registration and motion correction of brain images," Neuroimage 17(2), 825-841 (2002).

38. M. Jenkinson and S. Smith, "A global optimisation method for robust affine registration of brain images," Med. Image Anal. 5(2), 143-156 (2001).

39. S. Feng, F. A. Zeng, and B. Chance, "Photon migration in the presence of a single defect: a perturbation analysis," Appl. Opt. 34(19), 38263837 (1995).

40. L. Kocsis, P. Herman, and A. Eke, "The modified Beer-Lambert law revisited," Phys. Med. Biol. 51(5), N91-98 (2006).

41. D. Delpy et al., "Estimation of optical pathlength through tissue from direct time of flight measurement," Phys. Med. Biol. 33, 1433 (1988).

42. A. Duncan et al., "Optical pathlength measurements on adult head, calf and forearm and the head of the newborn infant using phase resolved optical spectroscopy," Phys. Med. Biol. 40(2), 295-304 (1995).

43. F. Scholkmann et al., "How to detect and reduce movement artifacts in near-infrared imaging using moving standard deviation and spline interpolation," Physiol. Meas. 31(5), 649-662 (2010).

44. M. Dehaes et al., "Assessment of the frequency-domain multi-distance method to evaluate the brain optical properties: Monte Carlo simulations from neonate to adult," Biomed. Opt. Express 2(3), 552-567 (2011).

45. T. Funane et al., "Quantitative evaluation of deep and shallow tissue layers' contribution to fNIRS signal using multi-distance optodes and independent component analysis," Neuroimage 85(Pt 1), 150165 (2014).

46. Q. Zhang, G. E. Strangman, and G. Ganis, "Adaptive filtering to reduce global interference in non-invasive NIRS measures of brain activation: how well and when does it work?" Neuroimage 45(3), 788-794 (2009).

47. G. Strangman et al., "A quantitative comparison of simultaneous BOLD fMRI and NIRS recordings during functional brain activation," Neuroimage 17(2), 719-731 (2002).

48. Y. Hoshi, N. Kobayashi, and M. Tamura, "Interpretation of near-infrared spectroscopy signals: a study with a newly developed perfused rat brain model," J. Appl. Physiol. 90(5), 1657-1662 (2001).

49. V. Toronov et al., "The roles of changes in deoxyhemoglobin concentration and regional cerebral blood volume in the fMRI BOLD signal," Neuroimage 19(4), 1521-1531 (2003).

Lia Maria Hocke is originally from Germany and is currently working as a postdoctoral fellow at the University of Calgary under Dr. Dunn. Since 2010 she worked under the supervision of Dr. Frederick and Dr. Tong at McLean Hospital affiliated with Harvard Medical School as part of her MS in clinical and cognitive neuroscience at Maastricht University and her PhD in biomedical engineering at Tufts. Her work focuses on multimodal acquisition and processing, specifically in combination with functional near infrared spectroscopy.

Kenroy Cayetano is a senior electrical engineer at McLean Hospital. $\mathrm{He}$ attended the Massachusetts Institute of Technology where he received his BS and MEng in electrical engineering with a minor in mechanical engineering. His thesis topic was on designing and modeling phased array coils. His current work includes RF coil design, board level circuitry and mechanical systems design. 
Hocke et al.: Optimized multimodal functional magnetic resonance imaging/near-infrared spectroscopy...

Yunjie Tong is an assistant professor in psychiatry, Harvard Medical School. He is originally from Beijing, China, where he received his BS degree in physics from Beijing University and his $\mathrm{PhD}$ in biomedical engineering from Tufts University. His thesis topic was on multimodal functional brain imaging and fast optical imaging of peripheral nerves. His current work includes concurrent functional MRI and functional near infrared spectroscopy and developments of new experimental and analytical methods in functional brain imaging.
Blaise Frederick is an associate professor in psychiatry, Harvard Medical School and a biophysicist at McLean Hospital. He received his $\mathrm{PhD}$ in biophysics from the University of California at Berkeley with Dr. Thomas Budinger. His training is in MR physics. He is the director of the McLean Opto-magnetic Group, and his research is focused on multimodal acquisition and processing for hemodynamic quantitation and physiological denoising of BOLD data. 